\title{
A comparative safety study of reported neurological adverse events with three COVID-19 vaccines
}

\author{
Mohit Sodhi ${ }^{1} \cdot$ Ali Samii ${ }^{2} \cdot$ Mahyar Etminan $^{3,4}$
}

Received: 26 September 2021 / Revised: 22 November 2021 / Accepted: 23 November 2021 / Published online: 9 January 2022

(c) The Author(s), under exclusive licence to Springer-Verlag GmbH Germany 2021

\section{Dear Sirs,}

Recently, numerous studies have alerted to the rare but clinically significant adverse events of some of the COVID-19 including cerebral venous sinus thrombosis (CVST) with the ChAdOx1/nCoV-19 (AstraZeneca) vaccine $[1,2]$.

Information on the safety of other clinically important neurological adverse events, especially potential differences between the most widely used vaccines such as the BNT162b2 (Pfizer-BioNTech), the mRNA-1273, (Moderna) and AstraZeneca vaccines, is scant.

We undertook a disproportionality analysis (DA) [3], a signal detection technique used by the Food and Drug Administration (FDA) to identify harmful adverse drug reaction profiles of different drugs [3]. DA compares the reported ADR of interest with the drug or vaccine under question to the identical outcomes with all other drugs without including the outcome for the drug in question. For signal quantification, one can compute a reported measure of effect, such as the reported odds ratio (ROR) [3]. We utilized VigiBase, which is the World Health Organization (WHO) database that reports potential adverse events of drugs worldwide. We sought to specifically quantify the reported events for cerebral venous sinus thrombosis (CVST) [1], ischemic stroke [4], Guillain-Barré syndrome [5], hemorrhagic stroke,

Mahyar Etminan

etminanm@mail.ubc.ca

1 Faculty of Medicine, University of British Columbia, Vancouver, Canada

2 Department of Neurology, University of Washington, Seattle, WA, USA

3 Deparmtment of Ophthalmology and Visual Sciences, Faculty of Medicine, The University of British Columbia, The Eye Care Center, Room 323-2550 Willow Street, Vancouver, BC V5Z 3N9, Canada

4 Department of Pharmacology and Therapeutics, Faculty of Medicine, University of British Columbia, Vancouver, Canada and transient ischemic attack (TIA) for three widely used COVID-19 vaccines including the AstraZeneca, Moderna, and Pfizer-BioNTech vaccines. We also computed RORs for the influenza vaccine utilized as a control vaccine.

All RORs are presented in Table 1. All four vaccines showed a signal for GBS with the AstraZeneca being the highest of the COVID-19 vaccines $(\mathrm{ROR}=2.74,95 \% \mathrm{CI}$ : 2.49-3.02) (Table 1). All three COVID-19 vaccines showed a signal for CVST and ischemic stroke. As expected, the AstraZeneca vaccine had the highest ROR for CVST $(R O R=15.43,95 \%$ CI: 13.73-17.34) (Table 1). The PfizerBioNTech (ROR $=4.40,95 \%$ CI: 3.56-5.44) and Moderna $(\mathrm{ROR}=2.67,95 \% \mathrm{CI}: 1.77-4.03)$ vaccines also showed an increase in reporting for CVST (Table 1). The PfizerBioNTech vaccine had the highest ROR for ischemic stroke $(\mathrm{ROR}=2.73,95 \% \mathrm{CI}: 2.48-3.01)$ and the AstraZeneca $(\mathrm{ROR}=1.38,95 \% \mathrm{CI}: 1.27-1.50)$ and Pfizer-BioNTech $(\mathrm{ROR}=1.24,1.13-1.36)$ vaccines both showed a signal for TIA (Table 1). The influenza vaccine was not associated with a higher rate of reporting for CVST, ischemic stroke, or TIAs (Table 1). No vaccine showed an increase in the reporting of hemorrhagic stroke (Table 1).

Our study demonstrates a high signal for CVST for the AstraZeneca vaccine as suggested by other studies [1]. We also found signals for CVST with the two mRNA vaccines, Pfizer-BioNTech and Moderna. The signal for ischemic stroke was also increased for all COVID-19 vaccines but not with the influenza vaccine, with the highest risk being with the Pfizer-BioNTech vaccine. Studies have shown that components of adenovirus-based vaccines, such as the adenovirus proteins, cause widespread platelet activation which can lead to thormbosis [6]. It is possible that mRNA vaccines cause a similar activation of platelets in the body, but to a lesser extent than adenovirus-based vaccines and thrombotic events may be specific to both mRNA and adenovirusbased COVID-19 vaccines. Only the Pfizer-BioNTech and AstraZeneca vaccines showed an increase in the reporting 
Table 1 Reported odds ratios and 95\% confidence intervals of neurological adverse events with the Influenza, AstraZeneca, Moderna, and Pfizer-BioNTech vaccines

\begin{tabular}{|c|c|c|c|c|}
\hline Adverse event & Influenza & AstraZeneca & Moderna & Pfizer-BioNTech \\
\hline Cerebral venous sinus thrombosis & $\begin{array}{l}{ }^{\mathrm{a}} 1 / 263,796 \\
\mathrm{~b}(0.06,0.01-0.46)\end{array}$ & $\begin{array}{l}351 / 393,877 \\
\mathbf{( 1 5 . 4 3 , 1 3 . 7 3 - 1 7 . 3 4 )}\end{array}$ & $\begin{array}{l}23 / 149,016 \\
(\mathbf{2 . 6 7}, \mathbf{1 . 7 7 - 4 . 0 3 )}\end{array}$ & $\begin{array}{l}91 / 357,991 \\
\mathbf{( 4 . 4 0 , 3 . 5 6 - 5 . 4 4 )}\end{array}$ \\
\hline Guillain-Barre syndrome & $\begin{array}{l}3618 / 263,796 \\
(\mathbf{5 3 . 9 6 , 5 1 . 8 0 - 5 6 . 2 1 )}\end{array}$ & $\begin{array}{l}428 / 393,877 \\
(\mathbf{2 . 7 4 ,} \mathbf{2 . 4 9 - 3 . 0 2 )}\end{array}$ & $\begin{array}{l}103 / 149,016 \\
(\mathbf{1 . 7 4}, \mathbf{1 . 4 3 - 2 . 1 2})\end{array}$ & $\begin{array}{l}217 / 357,991 \\
\mathbf{( 1 . 5 3 , 1 . 3 4 - 1 . 7 5 )}\end{array}$ \\
\hline Hemorrhagic stroke & $\begin{array}{l}10 / 263,796 \\
(0.14,0.07-0.25)\end{array}$ & $\begin{array}{l}58 / 393,877 \\
(0.53,0.41-0.69)\end{array}$ & $\begin{array}{l}30 / 149,016 \\
(0.72,0.50-1.04)\end{array}$ & $\begin{array}{l}82 / 357,991 \\
(0.82,0.66-1.02)\end{array}$ \\
\hline Ischemic stroke & $\begin{array}{l}27 / 263,796 \\
(0.24,0.16-0.35)\end{array}$ & $\begin{array}{l}358 / 393,877 \\
\mathbf{( 2 . 1 3 , 1 . 9 2 - 2 . 3 7 )}\end{array}$ & $\begin{array}{l}99 / 149,016 \\
(\mathbf{1 . 5 6}, \mathbf{1 . 2 8}-\mathbf{1 . 9 0})\end{array}$ & $\begin{array}{l}417 / 357,991 \\
\mathbf{( 2 . 7 3 , 2 . 4 8 - 3 . 0 1 )}\end{array}$ \\
\hline Transient ischemic attack & $\begin{array}{l}80 / 263,796 \\
(0.30,0.24-0.37)\end{array}$ & $\begin{array}{l}552 / 393,877 \\
(\mathbf{1 . 3 8}, \mathbf{1 . 2 7}-\mathbf{1 . 5 0})\end{array}$ & $\begin{array}{l}150 / 149,016 \\
(0.99,0.84-1.16)\end{array}$ & $\begin{array}{l}452 / 357,991 \\
(\mathbf{1 . 2 4}, \mathbf{1 . 1 3}-\mathbf{1 . 3 6})\end{array}$ \\
\hline
\end{tabular}

Bold values indicate significant signals

${ }^{a}$ Reported number of the ocular events for that vaccine/number of total reported events for that vaccine

${ }^{\mathrm{b}}$ Odds ratio, $95 \%$ confidence interval

of TIA and none of the vaccines showed an increased risk with hemorrhagic stroke.

GBS also showed a higher reporting rate across all vaccines which has been confirmed by other studies [7]. Given that all four vaccines in question in our study had a significantly elevated risk of GBS, it is possible that vaccineinduced GBS may be due to complex immune-mediated reactions that occur and may not be specific to the COVID19 vaccines per se.

Other studies have also examined adverse drug reactions secondary to COVID-19 vaccines. A meta-analysis of three clinical trials (two MRNA vaccines and one adenovirus) involving approximately 45,000 subjects examined adverse reactions related to the COVID-19 vaccines [8]. However, given the relatively short follow-up, the study could only examine acute adverse reactions such as fever, headache and fatigue [8]. Another study by Barda et al. used data from a large healthcare database from Israel and 884,828 subjects. This study did not find an increase in the risk of cerebrovascular accidents. However, the study participants were young adults possibly with a lower baseline risk of stroke (with an average age of 38) and older adults in long-term facilities as well as health care workers were excluded [9].

Our study has strengths and limitations. This is the first study that has quantified reported neurologic adverse events with three most utilized COVID-19 vaccines and use of the influenza vaccine as a control. The use of the influenza vaccine as a control enabled us to better understand whether the adverse events are specific to the COVID-19 vaccines. Two limitations of our study are that (1) DA does not allow for a causal assessment, such that a signal does not necessarily mean that the exposure and outcomes are related, and (2) all DAs are subject to reporting bias. However, it is unknown as to whether the reporting of these ADRs with the three vaccines is different. Due to the limitations of DA, the hypothesis generated from this study must be validated in large epidemiologic studies.

This study found a safety signal with several neurologic ADRs including CVST, GBS, and ischemic stroke not only with the AstraZeneca vaccine but also with Pfizer and Moderna vaccines. Given the absence of well-designed comparative safety studies on the neurologic adverse events of all three vaccines, this study can shed light on the disproportionate number of reports for important neurological adverse event with the three most used COVID-19 vaccines. Future epidemiologic studies are needed to validate these findings.

Author contributions ME had full access of the data and takes full responsibility of the data. ME and MS: concept and design; ME: acquisition, analysis, or interpretation of data; ME and MS: drafting of the manuscript; ME, MS, and AS: critical revision of the manuscript; ME, MS, and AS: intellectual content. Statistical analysis: Uppsala Monitoring Center; ME: supervision.

Funding Department of Ophthalmology and Visual Sciences, University of British Columbia.

\section{Declarations}

Conflicts of interest None of the authors have any conflicts to report. Information disclosed in this manuscript does not represent the opinion of the Uppsala Monitoring Center or the World Health Organization.

Ethical approval Ethics was not required to be obtained for this study.

\section{References}

1. Pottegård A, Lund LC, Karlstad $\emptyset$ et al (2021) Arterial events, venous thromboembolism, thrombocytopenia, and bleeding after vaccination with Oxford-AstraZeneca ChAdOx1-S in Denmark and Norway: population based cohort study. BMJ 373:n1114 
2. Health Canada. Health Canada issues label change on the AstraZeneca and COVISHIELD COVID-19 Vaccinations. https://healt hycanadians.gc.ca/recall-alert-rappel-avis/hc-sc/2021/75389aeng.php. Accessed 5 Jul 2021

3. Data Mining at FDA - White Paper. https://www.fda.gov/scien ce-research/data-mining/data-mining-fda-white-paper?fbclid= IwAR0OH8e519i3rLm9yrt3rMK9iF0eB1oqFvhb5181u55ZihYm TFzvuRfyLlM. Accessed 5 Jul 2021

4. Al-Mayhani T, Saber S, Stubbs MJ et al (2021) Ischaemic stroke as a presenting feature of $\mathrm{ChAdOx} 1 \mathrm{nCoV}-19$ vaccine-induced immune thrombotic thrombocytopenia. J Neurol Neurosurg Psychiatry 92(11):1247-1248

5. Maramattom BV, Krishnan P, Paul R et al (2021) Guillain-Barré Syndrome following ChAdOx1-S/nCoV-19 Vaccine. Ann Neurol 90(2):312-314

6. Greinacher A, Selleng K, Wesche J et al (2021) Towards understanding ChAdOx1 nCov-19 vaccine-induced immune thrombotic thrombocytopenia (VITT). Research Square (preprint). https://doi. org/10.21203/rs.3.rs-440461/v1

7. Patone M, Handunnetthi L, Saatci D et al (2021) Neurological complications after first dose of COVID-19 vaccines and SARS-CoV-2 infection. Nat Med. https://doi.org/10.1038/ s41591-021-01556-7

8. Amanzio M, Mitsikostas DD, Giovannelli F, Bartoli M, Cipriani GE, Brown WA (2021) Adverse events of active and placebo groups in SARS-CoV-2 vaccine randomized trials: a systematic review. Lancet Reg Health Eur 12: https://doi.org/10.1016/j. lanepe.2021.100253

9. Barda N, Dagan N, Ben-Shlomo Y et al (2021) Safety of the BNT162b2 mRNA COVID-19 vaccine in a nationwide setting. N Engl J Med 385(12):1078-1090 